SUPPORTING INFORMATION

\title{
Efficient Energy Conversion in Photochromic Ruthenium DMSO Complexes
}

Aaron A. Rachford, Jeffrey L. Petersen $\dagger$ and Jeffrey J. Rack*

Department of Chemistry and Biochemistry, Ohio University, Athens, OH 45701.

$\dagger$ C. Eugene Bennett Department of Chemistry, West Virginia University, Morgantown, WV 26506-6045 

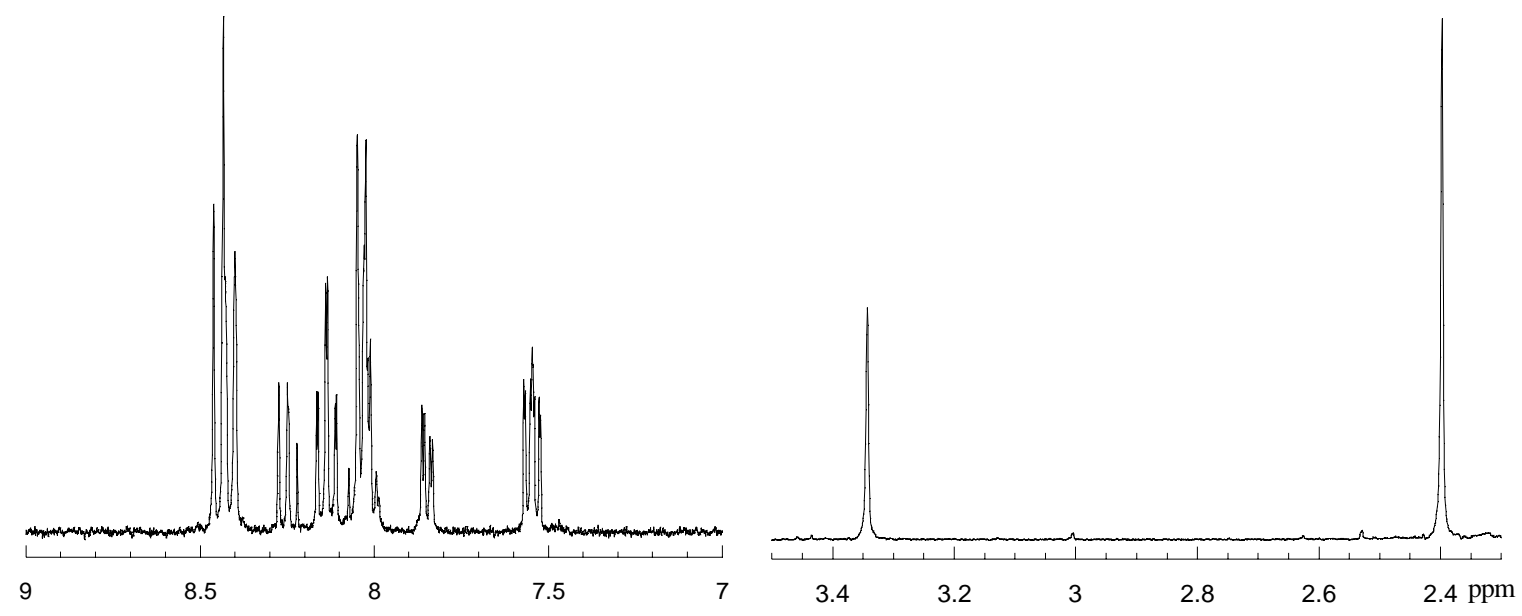

Figure S1. ${ }^{1} \mathrm{H}$ NMR of trans-[Ru(tpy)(Mepic)(dmso) ${ }^{+}(2)$ in $\mathrm{CD}_{3} \mathrm{CN}(\delta=3.34 \mathrm{ppm}, 3 \mathrm{H}$, Mepic $_{C_{3}} ; \delta=2.39$ ppm, $6 \mathrm{H}$, dmso) 


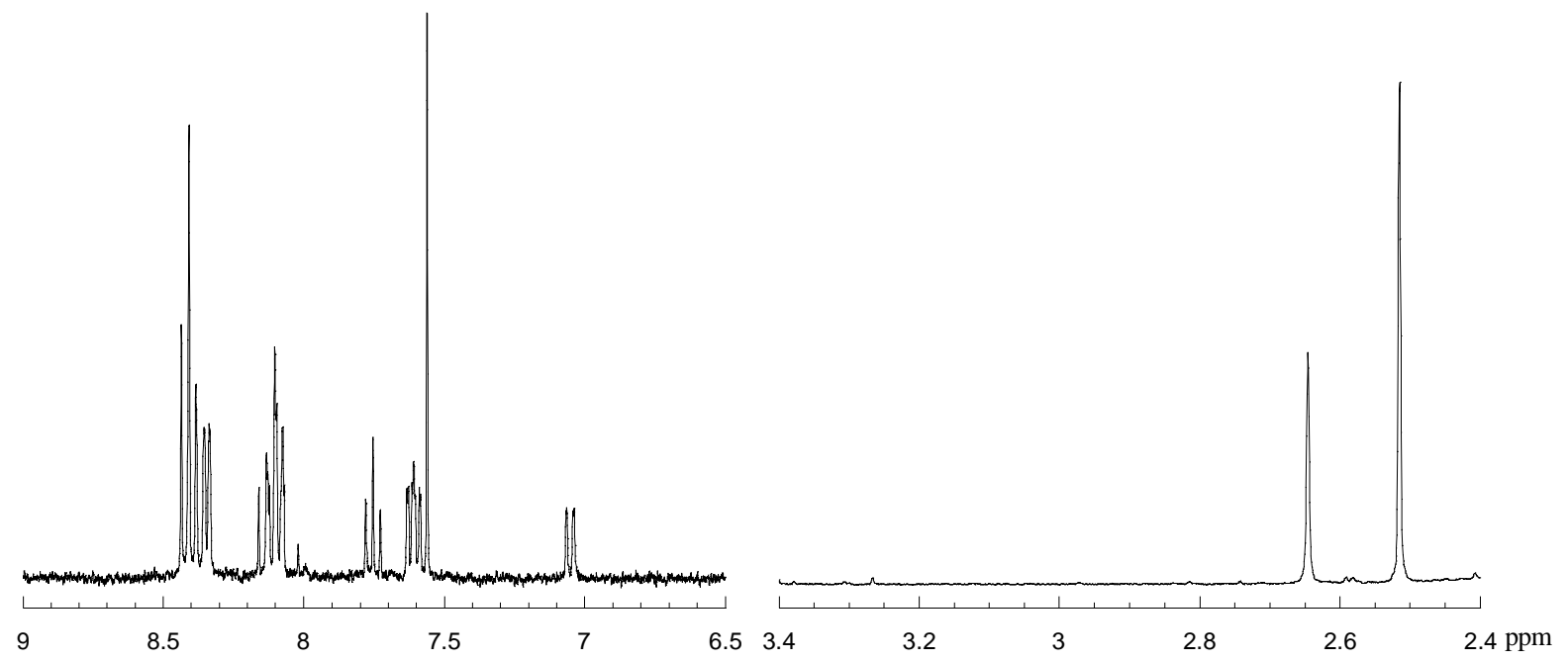

Figure S2. ${ }^{1} \mathrm{H}$ NMR of $c i s-[\mathrm{Ru}(\mathrm{tpy})(\mathrm{Mepic})(\mathrm{dmso})]^{+}(3)$ in $\mathrm{CD}_{3} \mathrm{CN}(\delta=2.64 \mathrm{ppm}, 3 \mathrm{H}$, Mepic $_{C_{3}} ; \delta=2.51$ ppm, 6H, dmso). 

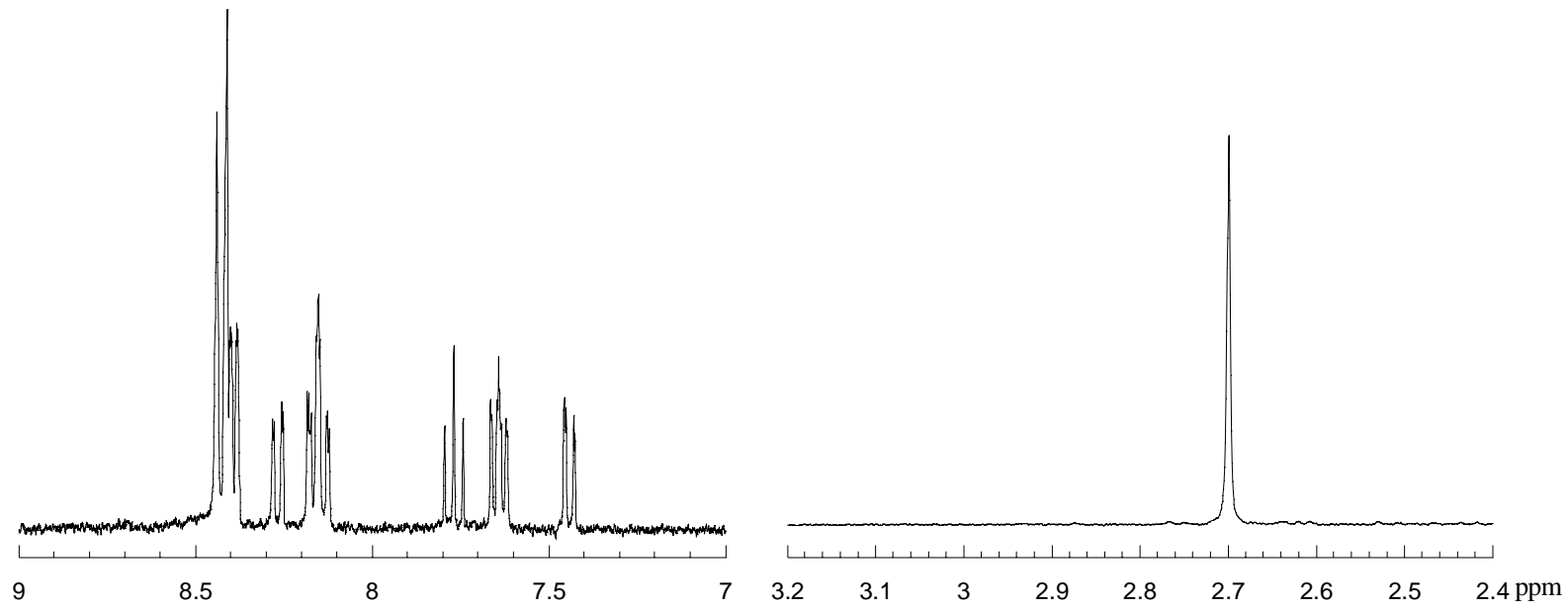

Figure S3. ${ }^{1} \mathrm{H}$ NMR of $c i s-[\mathrm{Ru}(\mathrm{tpy})(\mathrm{Brpic})(\mathrm{dmso})]^{+}(4)$ in $\mathrm{CD}_{3} \mathrm{CN}(\delta=2.70 \mathrm{ppm}, 6 \mathrm{H}$, dmso). 


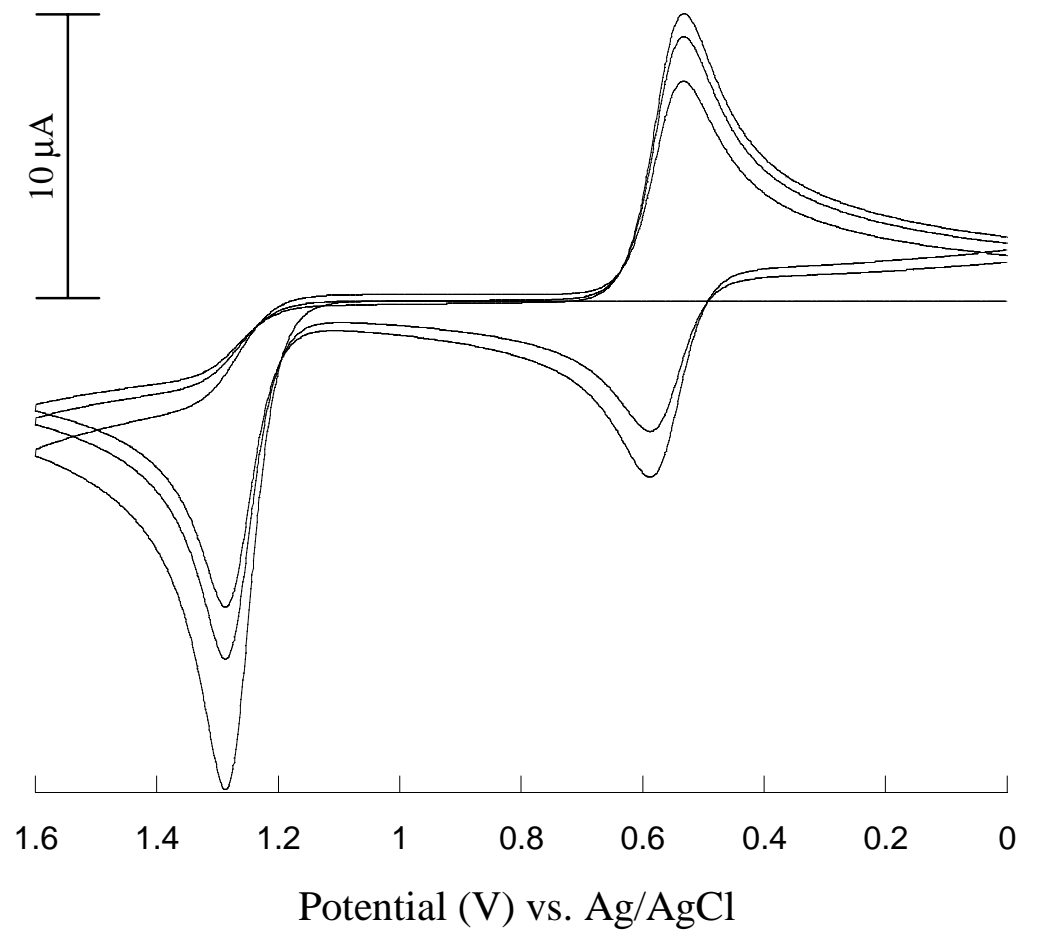

Figure S4. Simulated cyclic voltammogram of 2 generated from an ECEC mechanism. Simulation parameters: $\mathrm{E}^{\circ}(\mathrm{S})=1.30 \mathrm{~V}, \mathrm{E}^{\circ}(\mathrm{O})=0.56 \mathrm{~V}, \mathrm{k}_{\mathrm{S} \rightarrow \mathrm{O}}=50 \mathrm{~s}^{-1}$ on $\mathrm{Ru}(\mathrm{III}), \mathrm{k}_{\mathrm{O} \rightarrow \mathrm{S}}=$ $0.002 \mathrm{~s}^{-1}$ on $\mathrm{Ru}(\mathrm{II})$, scan rate $(\mathrm{v})=0.1 \mathrm{Vs}^{-1}$. 
Table S1. Selected Bond Distances and Angles for 4B.

\begin{tabular}{llll}
\hline distance & $(\AA)$ & angles & $(\mathrm{deg})$ \\
\hline Ru2-N5 & $2.077(3)$ & N5-Ru2-N7 & $159.4(2)$ \\
Ru2-N6 & $1.956(3)$ & N5-Ru2-N6 & $79.8(2)$ \\
Ru2-N7 & $2.072(3)$ & N6-Ru2-N7 & $79.8(1)$ \\
Ru2-N8 & $2.174(3)$ & N5-Ru2-S2 & $89.0(1)$ \\
Ru2-O4 & $2.092(3)$ & N6-Ru2-S2 & $88.8(1)$ \\
Ru2-S2 & $2.240(1)$ & N7-Ru2-S2 & $93.3(1)$ \\
S2-O6 & $1.472(3)$ & N8-Ru2-S2 & $167.9(1)$ \\
S2-C45 & $1.763(5)$ & O4-Ru2-S2 & $89.7(1)$ \\
S2-C46 & $1.774(4)$ & O6-S2-Ru2-O4 & 156.7 \\
\hline
\end{tabular}

